\title{
Article
}

\section{Looking back, looking forward: recovery journeys in a high secure hospital}

Mckeown, Mick, Jones, Fiona, Foy, Paul, Wright, Karen Margaret and Blackmon, Michael Oakes

Available at http://clok.uclan.ac.uk/12829/

Mckeown, Mick ORCID: 0000-0003-0235-1923, Jones, Fiona, Foy, Paul, Wright, Karen Margaret ORCID: 0000-0003-0693-7294 and Blackmon, Michael Oakes (2016) Looking back, looking forward: recovery journeys in a high secure hospital. International Journal Of Mental Health Nursing, 25 (3). pp. 234-242. ISSN 1445-8330

It is advisable to refer to the publisher's version if you intend to cite from the work. http://dx.doi.org/10.1111/inm.12204

For more information about UCLan's research in this area go to http://www.uclan.ac.uk/researchgroups/ and search for < name of research Group $>$.

For information about Research generally at UCLan please go to http://www.uclan.ac.uk/research/

All outputs in CLoK are protected by Intellectual Property Rights law, including Copyright law. Copyright, IPR and Moral Rights for the works on this site are retained by the individual authors and/or other copyright owners. Terms and conditions for use of this material are defined in the policies page.

\section{CLoK}

Central Lancashire online Knowledge www.clok.uclan.ac.uk

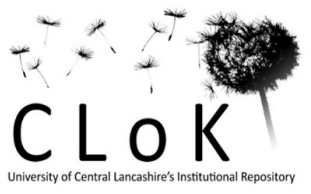


Looking back, looking forward: recovery journeys in a high secure hospital

\author{
McKeown, M., Jones, F., Foy, P., Wright, K., Paxton, T. \& Blackmon, M. \\ Accepted for publication 23.11.15 \\ International Journal of Mental Health Nursing
}

\begin{abstract}
A qualitative study of staff and service users' views of recovery was undertaken in a UK high secure hospital working to implement recovery practices. 30 staff and 25 service users participated in semi-structured interviews or focus groups. Thematic analysis identified four broad accounts of how recovery was made sense of in the high secure environment: the importance of meaningful occupation; valuing relationships; recovery journeys and dialogue with the past; and recovery as personal responsibility. These themes are discussed with an emphasis on service user strategies of cooperation or resistance, respectively advancing or impeding progress through the system. In this context the notion of cooperation is, for many, commensurate with compliance with a dominant medical model. The policy framing of recovery opens up contemplation of treatment alternatives, more participatory approaches to risk management, and emphasise the value of relational skills, but may not elude the overarching bio-psychiatric episteme.
\end{abstract}

\title{
Word count: 5000
}

\section{Introduction}

This paper presents findings from a qualitative study of service user and multi-disciplinary staff understandings of recovery within an English High Secure Hospital making concerted efforts to introduce recovery orientated approaches to care. Questions regarding what 'recovery' might mean in a high secure setting remain largely unresolved, but there is an interesting and growing body of research and development. Much of this has been advocated by the UK National Health Service endorsed program Implementing Recovery through Organisational Change (ImROC) (Drennan et al. 2014, Shepherd et al. 2010), and sustained by a national secure services recovery and outcomes network. The latter comprises nine regional networks supporting joint strategic and service development work between service users, staff and commissioners, and coordinated by an ex-service user now employed by Rethink Mental Illness, the mental health 
charity (see: http://www.recoveryandoutcomes.org/about-us/about-recovery-andoutcomes.html).

\section{Recovery practices in high secure hospitals}

Recovery has been explored as an organising concept within UK secure services, with interesting developments in all four high secure hospitals - Ashworth (Davies \& Abbot 2006, Chandley et al. 2014, Chandley \& Rouski 2014), Broadmoor (Adshead 2011, Adshead et al. 2015, Ayres et al. 2104, 2015, Drennan \& Aldred 2012, Dworkins \& Adshead 2011, Ferrito et al. 2012, Hayward \& Ayres 2011, Maguire \& Merrick 2012, Moore et al. 2012) and Rampton (Madders \& George 2014, Souter 2015, Völlm et al. 2014) in England and The State Hospital in Scotland (Laithwaite \& Gumley 2007, Laithwaite et al. 2009, Walker \& Langton 2012). In Ashworth, where this study was sited, initiatives include designated staff and service user recovery champions for each ward who also meet centrally for strategic purposes, widespread use of a collaborative recovery measure (the Recovery Star Secure - http://www.outcomesstar.org.uk/), implementation of an in-house recovery college, and an action research project focused upon recovery practices at ward level (Chandley \& Rouski 2014, Chandley et al. 2014). Practitioners at Ashworth and other secure units have assisted ImROC to produce practice development guidance (Drennan et al. 2014). Consequently, Chandley and colleagues (2014) at Ashworth suggest that the recovery concept can play an essential role in demonstrating the continuing relevance of high secure hospitals amidst various historical and contemporary criticisms of their legitimacy (see Pilgrim 2007).

\section{The meaning of recovery in a forensic context}

A small but growing literature focuses on recovery in forensic care (see Allen 2010, Ayres et al. 2014, 2015, Chandley \& Rouski 2014, Drennan \& Aldred 2012, Ferrito et al. 2012, Hayward \& Ayres 2011, Livingston et al. 2012, Mezey et al. 2010, Pouncey \& Lukens 2010, Simpson \& Penney 2011, Turton et al. 2011). Questions remain regarding what exactly 'recovery' means for people detained in high secure hospitals or how secure conditions impact the meaningfulness of recovery as an organising principle (Turton et al. 2011). At the heart of attempts to apply recovery models within forensic care are some quite fundamental ethical contradictions, such as confounding psychiatric recourse to notions of self-determination and criminal responsibility (Pouncey \& Lukens 2010). Previously, the vocabulary was of 'social rehabilitation' but the same competing forces of care and disciplinary regulation arguably persist (see Holmes 2000, Mason 
\& Mercer 1998). These concerns transcend the mere abstract, having very real implications for attempts at system change (Ramon 2011).

The juxtaposition of mental health problems with offender rehabilitation goals suggests that the aim of a recovery approach within forensic care is realisation of a meaningful life, or a life worth living' (Simpson \& Penney 2011: 304). Furthermore, any substantial recovery work must bridge concepts of therapy and security. Those engaged in offender therapy have remarked that for this group, who may have committed homicide, we are in the realms of thinking about 'life after death' (Adshead 2011, Adshead et al. 2015), with attention drawn to notions of personal 'redemption' (Ferrito et al. 2012). This means navigating tensions between a recovery orientation wishing to see the best in people and forensic cultures that acknowledge the worst in people; hence recovery practices in these settings must represent a sort of 'third way' approach (Dworkins \& Adshead 2011: 178).

Research in a medium secure setting showed the idea of recovery was rendered meaningful in terms of symptom reduction and feeling better about oneself, predicated upon medication, psychological support and positive effects of the secure environment (Mezey et al 2010). This is of course happily congruent with service expectations of the value of such institutional care and despite the avowed location of recovery as an alternative to simplistic medical models. Other studies in secure settings of, for instance, person centred care address similar issues of meaningful translation, understanding and appreciation as those more precisely defined by recovery (see Livingston et al. 2012, McKeown et al 2014).

For the mainly men who reside in high secure hospitals, at least one aspect of recovery is working to escape the identity of serious offender or killer, and this may be bound up with 'poignant expressions of regret and remorse' (Adshead et al. 2015: 79), or support from staff towards rediscovery of unique personhood, often alongside resisting the pull of institutionalising forces (Dunn 2014). Narrative therapy opens up opportunities for men who have killed to: 'discover (as opposed to recover) the "lived experience" of surviving a dreadful event which has been a tragedy' in different ways for both victims and perpetrators (Adshead et al. 2015: 79). This points to a number of complications associated with this client group and the environment within which care and treatment must take place: potential conflict between matters of personal empowerment and risk management, the importance of sense of safety (which could be provided or compromised by characteristics of the secure setting), the 
influence of relationships and social networks, and the value of work addressing identity and sense of self (Shepherd et al. 2015).

This study aimed to explore diverse viewpoints regarding how people make sense of recovery and experiences of recovery oriented assessment and treatment initiatives within the hospital.

\section{$\underline{\text { Methods }}$}

A purposive sample of 30 staff and 25 service users participated in either semi-structured interviews or focus groups. These were audiotaped and transcribed within the team. Potential participants were selected on the basis they exemplified different aspects of diversity, for example age, ward location, ethnicity, affinity or not for recovery initiatives. Reflecting the demographics of the hospital, all service users were male. To encourage as wide as possible participation, we did not collect information regarding service user participants' index offence, length of stay, or diagnosis. Rather, we restricted our interest to eliciting narratives of recovery and supporting factors from the perspective of participants. For the staff, we restricted ourselves to identification of participants' job role (see Figure 1).

In addition, we conducted a series of three focus groups with a core group of five service users with a specific aim of exploring key emergent themes in more depth. The majority of the data collection was undertaken by MM with the assistance of PF. Ethical approval was granted by the North West Research Ethics Committee and local hospital governance committee, and all participants' anonymity and confidentiality were assured. All data was subject to thematic analysis (Coffey \& Atkinson 1996). Data collection took place between November 2013 and March 2014.

A specially convened group of academics, practitioner staff and service users assisted with the planning and oversight of the project and provided a link into a larger 'recovery champions group' constituted for consultative and development purposes at the hospital. One of our research team, FJ, has her own previous experience of detention in high secure services, and made a key contribution in liaison with service users at the hospital and the recovery champions group in particular, assisting with service user participant recruitment, ongoing communication. Another of our team, PF, was drawn from Ashworth staff and also assisted greatly with staff and service user recruitment. No incentives were offered for participation and 
there were no explicit exclusion criteria. Only small numbers of staff and service users refused invitations to participate. All data collection was guided by a list of topics devised by the research team in consultation with the Recovery Champions group. This topic guide covered issues such as understandings of recovery concept, what it means to individuals in the context of their experiences in the hospital, supportive factors and impediments, influence of secure environment, and interest and involvement in particular recovery initiatives.

All of the team were involved in data analysis. Provisional thinking about identified themes and finalised themes were discussed at respective meetings of the Recovery Champions group, who verified the trustworthiness of these findings and offered further insights helpful in refining our analysis.

Findings

[insert Figure 1.]

Identified themes reflect different understandings of recovery and include: the importance of meaningful occupation; valuing relationships; recovery journeys and dialogue with the past; and recovery as personal responsibility. These are expanded upon below and illustrated with quotes from participants, all of which are from different individuals.

The importance of meaningful occupation

Service users and staff recognised the importance of meaningful occupation. This was currently organised in a central rehabilitation block, providing opportunities for leisure, education, or workshop activities. There was also provision of occupational activities on wards for individuals unable to leave. For those who got involved, keeping occupied was consistently pointed out as central to personal understandings of recovery and wellness:

I've got quite a busy schedule here and if I didn't, I know that keeps me ticking over. I need that to keep me going. Being busy is good. There's lads here that came in same time as me 
and they just sit around the ward every day. I'd end up in fights and falling out with people and start getting depressed. (service user)

Those involved in work related activity remarked that approximation to a 'work culture' had positive effects for service users beyond the acquisition of skills - including enhanced confidence and esteem and wellbeing. This was associated with positive commentary on the social-relational aspects of these activities, sometimes evidenced in looser inter-personal boundaries resulting in work-like 'banter' for instance:

On the work parties you can have banter, in ways that might not be allowed on the wards. I do appreciate the need for boundaries but think this is important for how we relate to each other as a work group. Its nearer to real life and that's important. (staff)

I think the occupations staff get it that we have work skills already or are getting them as we work. They treat us more as workers and we all do with each other. You can take the mick and have a laugh, just like you would in a real job. That's the best of it. (service user)

Opportunities to be part of work 'gangs' doing painting and decorating in the hospital opened up experiences of 'job satisfaction' and autonomy - for instance in choosing how one's own ward were to be decorated.

Valuing relationships

Relationship between staff and service users were seen as a crucial part of people's recovery trajectories:

Its all about the relationships with staff. I wouldn't even have started my recovery if I hadn't started to trust some staff, and this wouldn't have happened without them being ok 
with me. Yes, I'd say the relationships is the important bit. Nothing is going to happen without good relationships (service user).

We understand that relationships are crucial for recovery. In my view the effort to establish a therapeutic or supportive relationship, whatever you want to call it, is essential for anything we might want to achieve with these patients. (staff)

This isn't to say that all such relationships were seen positively. Indeed, the latter quote suggests a complicated disposition towards caring relationships. Rather, certain staff or key workers or, indeed, teams, were singled out as having made a critical difference, and the progress would be identified with the quality of inter-personal relationships; degrees of trust, communication styles, listening, and showing an interest in people's personal recovery.

The framing of trusting relationships was often grounded in mixed receptions of each other on the part of service users and staff, bound up with prior expectations and micro-cultures:

When I got to the ward, it put me on the back foot because they offered me a cup of tea and a sandwich. I couldn't trust them even when they were being nice. (service user)

For some, the Recovery Star was an important vehicle for supporting positive relationships, providing a set of openings to focus on individual recovery, or to think about routine care in these terms.

Receiving explanations for decisions was appreciated, this in the context of user involvement forum:

You go from thinking 'they're taking everything away from us' and we never get a say to having an explanation to take back to other patients.(service user) 
Cooperation was felt to be supported by staff taking time to explain decisions:

I can accept decisions better if I get a reason rather than being told 'no, we can't do that'. Other times, there's no explanation which is frustrating. (service user)

Many of the participants also pointed to the value of service users maintaining or repairing relationships with family and friends external to the hospital:

Families could be significant in the recovery journey by looking forward to extended support as well as professional support in the future when patients move on from a particular establishment (staff).

Recovery journeys and dialogue with the past

The majority of service users in the study were actively involved in recovery initiatives, some being identified as recovery champions. For these service users appreciative of recovery practices, the issue of cooperation to advance care plan goals towards achieving desirable outcomes was prominent. For the majority, this equated to agreement with medication and an illness framing of problems and needs:

It's all about me getting better. I see the point of the medication now. I've been really ill and now I'm moving on. (service user)

A small number of participants were much less cooperative, or resisted the system and were subject to coercive measures, such as segregation from other service users. At least for some, resistance was described in terms of rejection of medicalisation or diagnosis: 
That was one of the things I questioned and because I've been labelled with 'this' and because I was challenging something I didn't agree with, I was rebelling against how the system viewed me. (service user)

A few of the presently cooperative service users had, previously, been more recalcitrant and reported a process, often slow, of coming round to the idea of cooperating:

I had no hope for 4 years, I spent a long time in segregation, my mental state went from bad to worse. I've had a lot of time to think about what I've done in the past. I've had to make an effort and make a conscious decision to change (service user)

Interestingly, both individual service users and to some extent the institution, and staff therein, where engaged in a redemptive dialogue with the past. Service users highlighted efforts and desires to put their offence or mental health problems behind them, typically linked to a sense that this would better enable social acceptance and future reintegration to society or family:

I feel now I've turned things around. Got my life back. I want my family to get to know me properly now. How I am now, not what I was like before. (service user)

This would be related as hard, perhaps painful work and there was an ever-present dilemma over the depth of conversations or disclosures grounded in anxieties that the reaction of care teams might be to close off or slow down exit from the system:

It is [expletive] tough as [expletive]. Don't let anyone tell you that's easy. You have to do it but it's not easy. I don't think anyone really lets on [about] everything going on in their head. (service user) 
A variant on this narrative for some service users was the recollection that reflections about remorse, regret and guilt might be more likely to occur with ministers than clinicians, in a context of spiritual support.

Some of the looking back was in terms of reflecting on changing attitudes and disposition to the hospital and care regimes:

I didn't want to come. It was hospital. It was the loony bin. There was stigma about it. I told prison staff I didn't want to go but I'd already been accepted. (service user)

For staff involved in particular programmes of recovery work the language of recovery journeys was explicitly linked to notions of empowerment, holism and progress through the system, past and future. Not all service users were able to speak reflexively about their recovery, and for these there was more of a recitation of ticking off different referral opportunities (therapy groups for instance) without being able, or willing, to articulate the personal impact or whether they were ever involved in periods of introspective work on 'self'.

The hospital where the study took place had experienced two significant public inquiries in the 1990s and consequent organisational change. As such, many of the staff were also keen to distance themselves from the past, doing so by highlighting progressive developments, such as recovery initiatives:

This place, you know, has had a bad press over the years I've been here, to say the least. Now all the buzz around recovery puts that in perspective. We can look forward, I think. (staff)

For those service users actively involved in developing the hospital's programme of recovery work, there was a neat sense that this also contributed to personal recovery journeys: 
I've been involved in recovery star meetings to develop the recovery star. I was part of the development group. We have tailored it for high secure ourselves. I feel proud that I've been involved in creating the recovery star - it's a sense of achievement. (service user)

Recovery as personal responsibility

A number of service users and staff saw the capacity to demonstrate responsibility for one's own actions as core to successful recovery. Perhaps unsurprisingly in this high secure context, both staff and service user accounts were thus concerned with a balancing of risk and responsibility. For service users this was associated with desires for autonomy, especially concerning decision-making:

People need to be given responsibility as well for their everyday actions ... I think it is part of recovery. I'm sure you'd rather make up your own mind and think do this, this and this. You'd rather think about it and decide what you want to do rather than be told what to do. (service user)

Staff in particular, but also some service users, spoke of the tensions between a recovery orientated emphasis on matters of autonomy and voice on the part of individuals detained in high secure settings and the all-pervading necessities of risk management. For some staff, the turn toward an affinity for a recovery model was treated with scepticism, either because they felt such a notion had no place in these settings and with these patients, or because this presented too many challenges or dilemmas to be effectively translated into meaningful practice:

Don't get me wrong, I'm not against progress. But, before we get carried away with the whole recovery thing we have to remind ourselves why we are here in the first place. The priority is risk management. If recovery focused work makes people less risky, then all well and good. But let's be real. Too much of anything can be a bad thing, and the last thing we need is to take our eye off security. (staff) 
This group saw the recovery agenda as opening up the possibility for risk management failures. In the extreme, the cooperation of service users could not be trusted as authentic because manipulative and devious individuals would play the system to their advantage. An opposing account saw the potential for service users to see that taking more responsibility for behaviour, including previously risky behaviour could be part of making progress towards goals or discharge:

I think one of the benefits of thinking about recovery ideas is the prospect of getting the service users more involved in things like risk assessment. Surely that favours them beginning to realise what risk factors are and how to take more responsibility for behaviour. This is difficult, and it won't work for everyone, but we should try it. (staff)

\section{Discussion}

Recovery practices can be meaningful in high secure hospitals, despite various constraining factors (Adshead et al. 2015, Drennan \& Aldred 2012, Ferrito et al. 2012). A small number of firsthand accounts attest to challenges but also affirm a meaningful recovery trajectory is possible for people detained in such services (Chandley \& Rouski 2014, Coffey 2006, McKeown et al. forthcoming, Moore et al. 2012). The requirement for substantial security measures will always have an impact and necessitate some adaptations compared to less restrictive environments. That said, recovery approaches offer opportunities to look afresh at risk management, opening up possibilities for judicious contemplation enhanced service user agency in risk minimisation.

Personal framings include both recovery from mental health difficulties and previous offending behaviour, and typically refer to reclamation of positive self-hood in the face of stigma or public opprobrium. These struggles to reinvent identity are supported by staff modelling and sustaining constructive relationships, and availability of meaningful occupation that facilitates a work-related identity with associated workmate relationships. This quest for a more socially valued identity connects with previous theorising and practice development associated with the desirability of 'good lives' (Simpson \& Penney 2011). The most obvious illustration of this is in the journey metaphor, highlighting reflexivity between 'looking back and looking forwards'. Notably, such juxtapositions are also open to staff wishing to escape damage to professional identity associated with previously damning critiques of the hospital regime. Thus, 'recovery' 
works as a mechanism for both service user and staff redemption. Reflections involve a sense of both looking back, into personal histories and the chequered history of the institution, and looking forward, often, but not exclusively, with the sort of hope implied in standard definitions of recovery. Arguably, recovery represents both the antithesis of the received view of high secure regimes and, seemingly paradoxically, the strategic adoption of recovery in these institutions may be the 'key to progress' (Chandley et al. 2014: 205).

The connection between meaningful occupation and recovery goals has been long recognised, and is perhaps an essential factor in thinking around recovery colleges (Perkins et al. 2012). In high secure services the opportunity to leave the ward environment regularly to participate in workshop, leisure or educative activities is welcomed by some but not necessarily open to, or taken up by, all. Such opportunities can be seen as means for breaking up the institutional passage of time as well as aiding individual recovery journeys (Chandley 2007). The value for recovery combines learning of knowledge and skills with other more subtle impact upon identity and sense of self. These effects are arguably supported by the relational skills of relevant staff (Tapp et al. 2013) as much as their practical and technical expertise, and insight into these processes contributes to job fulfilment. In the context of employment-like activities, positive 'worker' identities and satisfactions are also open to participating service users, playing into appreciation for these practices. Benefits extend into focused activities aimed at preparation for employment beyond discharge from hospital (Völlm et al. 2014).

Off ward occupational interventions are currently subject to organisational restructurings within the high secure estate, arising from managerial concerns over a proportional lack of uptake in a context of budget pressures. A substantial organisational challenge will be to protect the value of occupational services, maximising participation whilst also ensuring that ward based activities are available for the reluctant participants or those individuals who for various reasons are unable to attend central facilities. Some of this thinking in Ashworth also needs to attend to matters of optimising team-work between technicians and therapists.

The value of warm, effective, consistent relationships and communication is evident in this study and is emphasised in published first-person accounts (Tapp et al. 2013). For example, the service user voice in the Chandley and Rouski (2014) co-authored paper, also located in Ashworth, notes how simple factors assist in building trust, such as staff doing what they say 
they are going to do, smiling, or offering explanations for refusals. This person also stresses the importance of the key recovery concept hopefulness, bound up with identified turning points on a personal journey, including coming to terms with the past and being able to envisage future goals beyond the hospital. These points correspond with the 'dialogue with the past' theme elicited in the present study.

The notion of cooperation, and its flip side, recalcitrance, is interesting and worthy of further investigation, particularly the need to explore more deeply the factors that obtain for individuals who turn away from resisting the system to cooperating. The simplest explanation for perceived shifts from resistance towards cooperative treatment alliances suggests a wellness trajectory or maturation process, with the former strongly evident in accounts that emphasised cooperation in terms of medication adherence. Typically, this is described in terms of the establishment of insight into either, or both, identification of one's problems and needs in terms of illness and recognition that cooperation with the system is a pre-requisite for progress towards eventual discharge. Arguably, cooperation in this context is inevitably bound up with limited choice and may very well be effectively compelled upon service users. Such circumstances can also potentiate staff mistrusting the sincerity of service users' concordance with prescribed treatment and prevailing care conditions.

Autobiographical accounts have stressed the importance of key staff, skilled in the relational aspects of care, who can live with and hold the distress and disturbance associated with recalcitrance to the point where they are able to capitalise upon changes in disposition towards a more cooperative engagement with care and treatment (McKeown et al. forthcoming).

Organisational forms that emphasise involvement and democratisation of decision making, such as therapeutic communities, are rarely found in high secure settings, but arguably have promise for supporting forms of cooperation and alliance desired under a more general recovery approach (Taylor et al. 2012). Similarly, the potential for models of peer support capitalising on expertise born out of experience have also been mooted in the high secure context (Madders \& George 2014). Chandley and colleagues (2014) remark that many nurses working in high secure settings have the necessary skills and experience to apply recovery approaches in this context, and that effective relational skills are majorly important in this regard, but there is also room for further learning and enhancement of these skills across care teams. 
The propensity to cooperate and achieve attendant benefits also connects with the reported valuing of occupational activities (see Tapp et al. 2013). For example, cooperation in planning and execution of work projects can model democratic decision making and tangibly demonstrate the utility of working together as a group to realise goals. In a creative context this point is powerfully emphasised by Maguire and Merrick's (2012) observations on involvement in a joint staff and service user band, The Strongmen, at Broadmoor high secure hospital. They remark upon the turbulence of musical differences, eventually resolved through a commitment to cooperation within the band. Interestingly, a service user band member, 'Barry', had maintained a 35 year refusal to accept a psychiatric explanation of his index offence, a stance which altered as a consequence of taking up a leadership role in the band. The following quote from Barry confirms the importance of recovery frames that are not singularly bio-medical, allow for personal agency, and are supported by positive staff relationships:

I, a patient, initiated the project and the staff backed me and accepted me as the leader of the band. Most projects are initiated by the staff who tell the patients what to do. The project was good for me as it helped me regain confidence in my leadership skills which are an integral part of my personality, which I am happy to say is now well on the way to a complete recovery (Barry, cited in Maguire \& Merrick 2012: 119)

One impediment to uptake and implementation of recovery ideals and practices amongst the majority staff group is the emphasis within nurse education of bio-medical understandings of care, and the more general subordination of all disciplines under psychiatry. Arguably, these tendencies are as prominent within the relatively isolated forensic sector as in the mainstream (Chandley et al 2014) though, as has been noted, much progress has been made in this context and practices around involvement and recovery can in some regards be superior in certain secure settings (McKeown et al. 2014).

But what then of the determined recalcitrant, who can languish subject to extreme coercive measures for lengthy periods? What if at least some of this recalcitrance is bound up with a legitimate, understandable rejection of medical explanations for offending behaviour? - with individuals caught up in a medicalised Catch-22. Ideally, further research could help develop more sophisticated understandings of service users' views in this regard, and perhaps point towards alternative interventions that do not frame all resistance as illegitimate and 
acknowledge the implicit coercion in compliance. Such research would likely be problematic to undertake, given that recalcitrant individuals may be equally ill-disposed to cooperating within the research process.

\section{Conclusions}

Innovations for recovery oriented practice are being enacted in high secure environments and, notwithstanding institutional imperatives of security and control, do have palpable meaning for service users and staff. Identification of a recovery journey for the institution, moving on from previous criticisms, reflect the more personal journeys of redemption that cooperative and engaged service users are embarked upon. In that sense we concur with Chandley and Rouski's (2014: 91) conclusions that, for high secure services, the recovery path is well worth taking, but 'if this is a journey there is a long way to go'. Despite a single hospital focus, the research has wider international appeal when considering the felt impact of secure environments upon recovery practices. Further research is needed to explore propensities for cooperation or resistance in the high secure context. 


\section{References}

Adshead, G. (2011). The life sentence: narrative group therapy with men who have killed. Group Analysis, 44, 1-21.

Adshead, G., Ferrito, M. \& Bose, S. (2015). Recovery after homicide: narrative shifts in therapy with homicide perpetrators. Criminal Justice and Behavior, 42 (1), 70-81.

Allen S. (2010). Our stories: moving on, recovery and well-being. London: South West London \& St Georges Mental Health Trust Forensic Services.

Ayres J, Fegan T, Noak J. (2014). The recovery orientation of patients and staff in a high secure hospital. Mental Health Practice, 17 (7), 20-24.

Ayres, J., Fegan, T., \& Noak, J. (2015). Measuring recovery orientation in a forensic high-secure hospital. British Journal of Mental Health Nursing, 4 (1), 26-30.

Chandley, M. (2007). Ashworth time. In Pilgrim, D. (ed) Inside Ashworth: Professional accounts of institutional life. (pp. 135-156). Oxford: Radcliffe Publishing.

Chandley, M., Cromar-Hayes, M., Mercer, D., Clancy, B., Wilkie, I., Thorpe, G. (2014). The development of recovery based nursing in a high-security hospital: nurturance and safe spaces in a dangerous world? Mental Health and Social Inclusion, 18 (4), 203-214.

Chandley, M. \& Rouski, M. (2014). Recovery, turning points and forensics: views from the ward in an English high secure facility. Mental Health and Social Inclusion, 18 (2), 83-91.

Coffey, A. \& Atkinson, P. (1996). Making sense of qualitative data. Thousand Oaks, CA: Sage.

Coffey, M. (2006). Researching service user views in forensic mental health: a literature review. The Journal of Forensic Psychiatry \& Psychology, 17 (1), 73-107. 
Davies, S. \& Abbot, P. (2006). Forensic rehabilitation. In: G. Roberts, S. Davenport, F. Holloway. \& T. Tattan. (Eds), Enabling recovery: the principles and practice of rehabilitation psychiatry (pp. 351-368). London: Royal College of Psychiatry Publications.

Drennan G, Aldred D. (2012). Secure recovery: approaches to recovery in secure mental health settings. London: Routledge.

Drennan, G. \& Wooldridge, J. with Aiyegbusi, A., Alred, D., Ayres, J., Barker, R., Carr, S., Euson, S., Lomas, H., Moore, E., Stanton, D. \& Shepherd, G. (2014). Making Recovery a Reality in Forensic Settings. [ImROC briefing paper 10]. London: Centre for Mental Health/Mental Health Network/NHS Confederation.

Dunn, W. (2014). Book Review: Secure recovery: approaches to recovery in forensic mental health settings. Journal of Forensic Psychiatry \& Psychology, 25, 238-239.

Dworkins, E. \& Adshead, G. (2011). Working with offenders: challenges to the recovery agenda. Advances in Psychiatric Treatment, 17, 178-187.

Ferrito M, Vetere A, Adshead G, Moore E. (2012). Life after homicide: accounts of recovery and redemption of offender patients in a high secure hospital. Journal of Forensic Psychiatry \& Psychology, 23, 322-344.

Hayward, S. \& Ayres, J. (2011). A service evaluation of recovery support from the patient's perspective. British Journal of Wellbeing, 2 (5), 26-31.

Holmes, D. (2000). Governing the captives: forensic psychiatric nursing in corrections. Perspectives in Psychiatric Care, 41 (1), 3-13.

Laithwaite, H. \& Gumley, A. (2007). Sense of self, adaptation and recovery in patients with psychosis in a forensic NHS setting. Clinical Psychology \& Psychotherapy, 14, 302-316. 
Laithwaite, H., O'Hanlon, M., Collins, P., Doyle, P., Abraham, L., Porter, S. \& Gumley, A. (2009). Recovery after psychosis (RAP): A compassion focused programme for individuals residing in high security settings. Behavioural and Cognitive Psychotherapy, 37, 511-526.

Livingston J, Nijdam-Jones A, Brink J. (2012). A tale of two cultures: examining patient-centred care in a forensic mental health hospital. Journal of Forensic Psychiatry and Psychology, 23, 345360.

Madders, S. \& George, C. (2014). "I couldn't have done it on my own." Perspectives of patients preparing for discharge from a UK high secure hospital. Mental Health Review Journal, 19 (1), 27-36.

Maguire, A. \& Merrick (2012). Walking the line: music therapy in the course of the recovery approach in a High Secure Hospital. In: S. Compton Dickinson, H. Odell-Miller \& J. Adlam (Eds), Forensic Music Therapy: A Treatment for Men and Women in Secure Hospital Settings (pp. 104120). London: Jessica Kingsley.

Mason, T. \& Mercer, D. (1998). Critical perspectives in forensic care: inside out. Basingstoke: Macmillan.

McKeown, M., Jones, F. \& Callaghan, I. (forthcoming). Services for people requiring secure forms of care. In: M. Chambers (Ed), Psychiatric and Mental Health Nursing: the craft of caring. third edition London: Hodder Arnold.

McKeown, M., Jones, F., Wright, K., Spandler, H., Wright, J., Fletcher, H., Duxbury, J., McVittie, J., Simon. \& Turton, W. (2014). It's the talk: a study of involvement practices in secure mental health services. Health Expectations, DOI: 10.111/hex.12232 
Mezey G, Kavuma M, Turton P, Demetriou A, Wright C. (2010). Perceptions, experiences and meanings of recovery in forensic psychiatric patients. The Journal of Forensic Psychiatry and Psychology, 21, 683-696.

Moore, E., Lumbard, D., Carthy, J., Ayres, J., 19 Hospital residents and one former patient (2012). Giving voice to recovery: perspectives from within a high secure hospital. In: G. Drennan \& D. Aldred (Eds), Secure recovery: approaches to recovery in secure mental health settings (pp. 73-87). London: Routledge.

Perkins, R., Repper, J., Rinaldi, M., \& Brown, H. (2012). 1. Recovery Colleges. [ImROC briefing paper]. London: Centre for Mental Health.

Pilgrim, D. (2007). Ashworth in context. In D. Pilgrim (Ed), Inside Ashworth: professional reflections of institutional life (pp. 1-21) Oxford: Radcliffe.

Pouncey C. \& Lukens J. (2010). Madness versus badness: the ethical tension between the recovery movement and forensic psychiatry. Theoretical Medicine \& Bioethics, 31, 93-105.

Ramon, S. (2011). Organisational change in the context of recovery-oriented services. The Journal of Mental Health Training, Education and Practice, 6 (1), 38-46.

Shepherd, A., Doyle, M., Sanders, C. \& Shaw, J. (2015). Personal recovery within forensic settings: systematic review and meta-synthesis of qualitative studies. Criminal Behaviour and Mental Health, DOI: 10.1002/cbm.1966

Shepherd, G., Boardman, J. \& Burns, M. (2010). Implementing recovery. A methodology for organisational change. London: Sainsbury Centre for Mental Health.

Simpson, A. \& Penney, S (2011). The recovery paradigm in forensic mental health services. Criminal Behaviour and Mental Health, 21 (5), 299-306.

Souter, G. (2015). Evaluating the named nurse understanding of recovery in forensic mental health. British Journal of Mental Health Nursing, 4 (2), 72-76. 
Tapp, J., Warren, F., Fife-Schaw, C., Perkins, D. \& Moore, E. (2013). What do the experts by experience tell us about 'what works' in high secure forensic inpatient hospital services?. The Journal of Forensic Psychiatry \& Psychology, 24 (2), 160-178.

Taylor, J., Trout, S., Christopher, J. \& Bland, A. (2012). A therapeutic community for personality disorder in a high secure intellectual disability service: inception and early experiences. Advances in Mental Health and Intellectual Disabilities, 6 (4), 165-170.

Turton P, Demetriou A, Boland W, Gillard S, Kavuma M, Mezey G, Mountford V, Turner K, White S, Zadeh E, Wright C. (2011). One size fits all: or horses for courses? Recovery-based care in specialist mental health services. Social Psychiatry and Psychiatric Epidemiology, 46 (2), 127 136.

Völlm, B., Panesar, K. \& Carley, K. (2014). Promoting work-related activities in a high secure setting: exploration of staff and patients' views. The Journal of Forensic Psychiatry \& Psychology, 25 (1), 26-43.

Walker, H. \& Langton, D. (2012). Recovery evaluation: the Scottish Forensic Services. In: G. Drennan \& D. Aldred (Eds), Secure recovery: approaches to recovery in secure mental health settings (pp. 202-216). London, UK: Routledge. 